\title{
ON RINGS OF WHICH ANY ONE-SIDED QUOTIENT RINGS ARE TWO-SIDED
}

\author{
Y. UTUMI
}

0. Introduction and preliminaries. Following R. E. Johnson [1] we assume in this paper that any rings we shall be concerned with satisfy either one or both of the following conditions:

$\left(J_{l}\right)$ If the right annihilator of a left ideal $A$ is nonzero, then there exists a nonzero left ideal $B$ such that $A \cap B=0$.

$\left(J_{r}\right)=$ the right left symmetry of $\left(J_{l}\right){ }^{1}$

We say that a ring is a $J_{l}$-ring, a $J_{r}$-ring or a $J$-ring if it satisfies $\left(J_{l}\right),\left(J_{r}\right)$ or both of them.

A module $A$ is called an essential extension of a submodule $B$ if $B \cap C \neq 0$ for every nonzero submodule $C$ of $A$. A module is said to be injective if it is a direct summand of every extension module. It is well known that every module $M$ has a maximal essential extension $\hat{M} . \hat{M}$ is injective, and is unique to within an isomorphism over $M$.

Let $S$ be a $J_{l}$-ring. Then we can define the multiplication in the maximal essential extension $\hat{S}$ of the left $S$-module $S$ such a way that (i) $\hat{S}$ forms a ring and (ii) the multiplication coincides, on $S \times \hat{S}$, with the scalar multiplication. This ring is unique up to an isomorphism over $S$, and is denoted by $\bar{S}_{l}$. As is known, $\bar{S}_{l}$ is regular (in the sense of von Neumann), and is left self injective, that is, injective as a left module over itself. An extension ring $T$ of a $J_{l}$-ring $S$ is called a left quotient ring of $S$ if the left $S$-module $T$ is an essential extension of the left $S$-module $S$. It is also known that every left quotient ring of $S$ is isomorphic, over $S$, to a subring of $\bar{S}_{l}$. Thus, $\bar{S}_{l}$ is the maximal left quotient ring of $S$.

We define similarly a right quotient ring and the maximal right quotient ring $\bar{S}_{r}$ of a $J_{r}$-ring $S$.

For any $J$-ring $S$ it is easily seen that the following conditions are equivalent:

(i) There exists an extension ring $T$ of $S$ with the properties that (a) it is regular (both left and right) self injective, and (b) every nonzero one-sided $S$-submodule of $T$ has a nonzero intersection with $S$.

(ii) Every left quotient ring of $S$ is a right quotient ring of $S$, and every right quotient ring of $S$ is a left quotient ring of $S$.

In this case any maximal left quotient ring of $S$ and any maximal

Received by the editors January 13, 1962.

1 In the terminology of Johnson [1] $\left(J_{l}\right)\left(\left(J_{r}\right)\right)$ means that the left (right) singular ideal of the ring is zero. 
right quotient ring of $S$ are mutually isomorphic over $S$. We denote this fact by writing $\bar{S}_{l}=\bar{S}_{r}$.

The main theorem (Theorem 3.3) in this paper states that for any $J$-ring $S$ we have $\bar{S}_{l}=\bar{S}_{r}$ if and only if $S$ satisfies the converse of $\left(J_{l}\right)$ and $\left(J_{r}\right)$, that is, the following two conditions:

$\left(K_{l}\right)$ If $A \cap B=0, B \neq 0$, for left ideals $A$ and $B$, then the right annihilator of $A$ is nonzero;

$\left(K_{r}\right)=$ the right left symmetry of $\left(K_{l}\right)$.

1. Strongly regular rings. A ring is called strongly regular if for any element $x$ there exists an element $y$ such that $x^{2} y=x$. As is well known, in this case $x y$ is central idempotent and $x y=y x$. Every onesided ideal of a strongly regular ring is two-sided. A regular ring is strongly regular if and only if it is of index 1 , that is, it contains no nonzero nilpotent elements.

A left ideal $A$ of a ring is said to be closed if there are no left ideals $B$ such that $A \neq B$ and $B$ is an essential extension of $A$. The set of all closed left ideals of a $J_{l}$-ring $S$ forms a complete complemented modular lattice, which is denoted by $L(S)$. If a ring $T$ is a left quotient ring of a $J_{l}$-ring $S$, then $T$ is also a $J_{l}$-ring, and $L(T)$ is isomorphic to $L(S)$ by the correspondence $A(\in L(T)) \rightarrow A \cap S$. A left ideal of $S_{\imath}$ is closed if and only if it is principal. Thus, $L\left(\bar{S}_{l}\right)$ is the lattice of all principal left ideals of $\bar{S}_{l}$. Let $A, B \in L(S)$. Then $A \cap B$ is the intersection of $A$ and $B$, while $A \cup B$ is the maximal essential extension of $A+B$.

We can define similarly the notion of a closed right ideal. $R(S)$ denotes the lattice of all closed right ideals of a $J_{r}$-ring $S . R\left(\bar{S}_{r}\right)$ is the lattice of principal right ideals of $\bar{S}_{\boldsymbol{r}}$.

THEOREM 1.1. Let $S$ be a regular ring. Then the following conditions are equivalent:

(i) $S$ is strongly regular.

(ii) $L(S)$ is distributive.

(iii) The lattice $L$ of principal left ideals of $S$ is distributive.

Proof. (i) $\Rightarrow$ (ii). Let $A, B \in L(S)$. Then $A B \subset A \cap B=(A \cap B)^{2}$ $\subset A B$, and so $A B=A \cap B$. Thus, for any $P, Q, R \in L(S)$ we have $P \cap(Q+R)=P(Q+R)=P Q+P R=(P \cap Q)+(P \cap R)$. Since $L(S)$ is complemented, $P \cap(Q \cup R)=((P \cap Q) \cup(P \cap R)) \cup C$ and $((P \cap Q) \cup(P \cap R)) \cap C=0$ for some $C \in L(S)$. Then $((P \cap Q)$ $+(P \cap R)) \cap C=0$ and $P \cap(Q+R) \cap C=0$, hence $P \cap(Q \cup R) \cap C=0$, which means that $C=0$ and $P \cap(Q \cup R)=(P \cap Q) \cup(P \cap R)$.

(ii) $\Rightarrow$ (iii) follows immediately since $L$ is a sublattice of $L(S)$.

(iii) $\Rightarrow$ (i). Let $S e=S f, e=e^{2}$ and $f=f^{2}$. Then $S(1-e)=S(1-f)$ by (iii). Thus $e=f$. This shows that every idempotent is central. There- 
fore $x y x=x$ means $x^{2} y=x$, as desired. In case $S \boxplus 1$, it follows from the above argument $t S t$ is strongly regular for any $t=t^{2}$. Let $S \ni x$, and let $a, b, c$ be idempotents such that $S x=S a, x S=b S$ and $a S+b S=c S$. Then $x \in t S t, t=t^{2}$ for $t=a+c-a c$. Therefore $S$ is strongly regular.

COROLlaRy 1.2. If $S$ is strongly regular, then so is $\bar{S}_{l}$.

In fact, $L(S)$ is distributive by assumption. Since $L(S) \simeq L\left(\bar{S}_{\imath}\right)$, $L\left(\bar{S}_{l}\right)$ also is distributive, and hence $\bar{S}_{l}$ is strongly regular.

COROLlaRY 1.3. Let $S$ be a $J_{l}$-ring. Then $\bar{S}_{l}$ is strongly regular if and only if $L(S)$ is distributive.

In fact, $L\left(\bar{S}_{l}\right)$ is distributive if and only if so is $L(S)$.

THEOREM 1.4. Let $S$ be a J-ring. If $S$ has a strongly regular left quotient ring $P$ and a strongly regular right quotient ring $Q$, then $\bar{S}_{l}=\bar{S}_{r}$.

Proof. Let $0 \neq p \in P$. Then $a p=b \neq 0$ for some $a, b \in S$, and $a^{2} q=a$ for some $q \in Q$. Since $b \neq 0,0 \neq b^{2}=b a p$ and $b a \neq 0$. Hence $0 \neq(b a)^{2}$ $=b(a b) a$ and $0 \neq a b=a^{2} q b=a(a q) b=a(a q)^{2} b=a^{2}(q a q) b=a^{2}\left(a q^{2}\right) b$, whence $a q^{2} b \neq 0$. Thus, $\left(a q^{2} b\right) c=d \neq 0$ for some $c, d \in S$. $a b c=\left(a^{2} q\right) b c$ $=a(a q) b c=a(a q)^{2} b c=a^{2}(q a q b) c=a^{2}\left(a q^{2} b\right) c=a^{2} d$, and so $a^{2} p c=a(a p) c$ $=a^{2} d$. Hence $a^{2}(p c-d)=0$ and $\left((p c-d) a^{2}\right)^{2}=0$, which implies that $(p c-d) a^{2}=0$ and $p\left(c a^{2}\right)=d a^{2}$. Now $0 \neq d=a q^{2} b c=(a q)(q b c)$ $=(a q)^{8}(q b c)=(a q)(q b c)(a q)^{2}=\left(a q^{2} b c\right) a^{2} q^{2}=d a^{2} q^{2}$, and hence $d a^{2} \neq 0$. This shows that $P$ is a right quotient ring of $S$. Thus we have proved that every strongly regular left quotient ring of $S$ is a right quotient ring of $S$ provided $S$ has at least one strongly regular right quotient ring. It is not too hard to see that $\bar{S}_{l}$ is the maximal left quotient ring of $P$. Hence $S_{l}$ is strongly regular by Corollary 1.2 . Therefore $\bar{S}_{l}$ is a right quotient ring of $S$. This implies that every left quotient ring of $S$ is a right quotient ring of $S$. Similarly we can show that every right quotient ring of $S$ is a left quotient ring of $S$. Thus, $\bar{S}_{l}=\bar{S}_{r}$, completing the proof.

2. The condition $(K)$. For any subset $Z$ of an extension ring of a ring $S$ we denote by $l(S, Z)$ the set of all $x \in S$ such that $x Z=0$. Similarly $r(S, Z)$ denotes the right annihilator, in $S$, of $Z$.

Lemma 2.1. Let $S$ be a $J_{l}$-ring, and let $A \in L\left(\bar{S}_{l}\right)$. Then $r(S, A \cap S)$ $=r(S, A)$.

Proof. $\supset$ is obvious. Let $(A \cap S) x=0, x \in S$, and set $B=l\left(\bar{S}_{l}, x\right)$. Since $B \in L\left(\bar{S}_{l}\right)$ and $B \cap S \supset A \cap S$, we have $B \supset A$ by the isomorphism $L(S) \simeq L\left(\bar{S}_{l}\right)$. Hence $A x=0$ and $x \in r(S, A)$. Therefore $r(S, A \cap S) \subset r(S, A)$. 
TheOREM 2.2. Let $S$ be a $J_{l}$-ring. Then the following conditions are equivalent:

$\left(K_{l}\right)$ If $A \cap B=0, B \neq 0$ for two left ideals $A$ and $B$, then the right annihilator of $A$ is nonzero.

$\left(K_{i}^{\prime}\right)$ Every closed left ideal is an annihilator.

$\left(K_{l}^{\prime \prime}\right)$ Every nonzero right ideal of $\bar{S}_{l}$ has a nonzero intersection with $S$.

Proof. $\left(K_{l}\right) \Rightarrow\left(K_{l}^{\prime \prime}\right)$. Suppose that $S \cap D=0$ for some nonzero right ideal $D$ of $\bar{S}_{l}$. Let $0 \neq e=e^{2} \in D$. By Lemma $2.1 r\left(S, \bar{S}_{l}(1-e) \cap S\right)$ $=r\left(S, \bar{S}_{l}(1-e)\right)=r\left(\bar{S}_{l}, \bar{S}_{l}(1-e)\right) \cap S=e \bar{S}_{l} \cap S \subset D \cap S=0$. Since $\left(\bar{S}_{l}(1-e) \cap S\right) \cap\left(\bar{S}_{l} e \cap S\right)=0$ and $\bar{S}_{l} e \cap S \neq 0$, this contradicts $\left(K_{l}\right)$.

$\left(K_{l}^{\prime \prime}\right) \Rightarrow\left(K_{l}^{\prime}\right)$. Let $A$ be a closed left ideal, and set $l(S, r(S, A))$ $=B$. If $A \neq B$, there is a left ideal $C \neq 0$ such that $B \supset C$ and $A \cap C=0$. Let $D$ be a maximal left ideal such that $D \supset A$ and $D \cap C=0$. Then $D$ is closed, and $D=\bar{S}_{l} e \cap S$ for some $e=e^{2} \neq 1$. By $\left(K_{l}^{\prime \prime}\right)(1-e) \bar{S}_{l} \cap S$ $\neq 0$. Let $0 \neq x \in(1-e) \bar{S}_{l} \cap S$. Then $D x=0$ and $A x=0$, hence $B x=0$ and $C x=0$. Thus $(D \oplus C) x=0$, and $(D \oplus C) \cap E=0$ for some left ideal $E \neq 0$. Hence $C \cap(D \oplus E)=0$, which contradicts the maximality of $D$. Therefore $A=B$.

$\left(K_{l}^{\prime}\right) \Rightarrow\left(K_{l}\right)$. Let $A \cap B=0$ and $B \neq 0$ for left ideals $A, B$ of $S$. Let $C$ be a maximal left ideal such that $C \supset A$ and $C \cap B=0$. Then $C$ is closed, and hence is an annihilator by $\left(K_{l}^{\prime}\right)$. If $r(S, A)=0$, then $r(S, C)=0$ and $C=S$, which means that $B=0$, a contradiction. Thus $r(S, A) \neq 0$, as desired.

CoRollary 2.3. $\bar{S}_{l}$ satisfies $\left(K_{l}\right)$ for any $J_{l}$-ring $S$.

In fact, $\bar{S}_{l}$ satisfies $\left(K_{l}^{\prime \prime}\right)$ because $\bar{S}_{l}$ is the maximal left quotient ring of itself. ${ }^{2}$

Corollary 2.4. If a J-ring $S$ satisfies $\left(K_{l}\right)$ and $\left(K_{r}\right)$, then $L(S)$ is dually isomorphic to $R(S)$ by the annihilator relation.

This is evident from $\left(K_{l}^{\prime}\right)$ and its symmetry. ${ }^{3}$

CoRollary 2.5. Let $S$ be a $J_{l}$-ring, and $T$ a left quotient ring of $S$.

(i) If $S$ satisfies $\left(K_{l}\right)$, then so does $T$.

(ii) If $T$ is a right quotient ring of $S$, and if $T$ satisfies $\left(K_{l}\right)$, then $S$ also satisfies it.

In fact, $\bar{S}_{l}$ is the maximal left quotient ring of $T$. Let $A$ be a nonzero right ideal of $\bar{S}_{l}$. If $S$ satisfies $\left(K_{l}\right), A \cap S \neq 0$ by $\left(K_{l}^{\prime \prime}\right)$. Hence

2 Of course, $\left(K_{l}\right)$ itself also follows immediately from the injectivity of $\boldsymbol{S}_{l}$.

It should be noted that every annihilator left (right) ideal of a $J_{l}-\left(J_{r}-\right)$ ring is closed. 
$A \cap T \neq 0$, and $T$ also satisfies $\left(K_{\imath}^{\prime \prime}\right)$. Conversely, if $T$ is a right quotient ring of $S, A \cap T \neq 0$ implies that $A \cap S \neq 0$. This proves (ii).

Lемма 2.6. Let $S$ be a $J_{l}$-ring, and let $S=A \oplus B, A$ and $B$ being ideals. If $S$ satisfies $\left(K_{l}\right)$, then so does $A$.

Proof. Let $P, Q$ be left ideals of $A$ such that $P \cap Q=0, Q \neq 0$. Then $(P \oplus B) \cap Q=0$. Hence $(P \oplus B)(a+b)=0, a+b \neq 0$ for some $a \in A$, $b \in B$. Therefore $S b=0$ and $b=0$, whence $a \neq 0$. Since $P a=0$, this proves that $A$ satisfies $\left(K_{l}\right)$.

3. The main theorem. A right ideal $A$ of $S$ is called large if $A \cap B$ $\neq 0$ for every right ideal $B \neq 0$.

Lemma 3.1. Let $S$ be a $J_{r}$-ring, and $T$ an extension ring. Denote by $U$ the set of all elements $x$ of $T$ such that $\{y \mid y \in S, x y \in S\}$ is a large right $i d e a l$ of $S$. If every nonzero left ideal of $T$ has a nonzero intersection with $S$, then $U$ is a right quotient ring of $S$.

Proof. It is easily seen that $U$ forms a subring of $T$. Let $V$ be the set of all elements $y \in T$ such that $r(S, y)$ is large. Then $V$ is a left ideal of $T$, and $V \cap S=0$ since $S$ is a $J_{r}$-ring. Hence $V=0$ by the assumption. It follows from this that $U$ is a right quotient ring of $S$, as desired.

We proved in [3] the following

TheOREM 3.2. Every regular left self injective ring $S$ is decomposed into the direct sum of two ideals $A$ and $B$ in such a way that $A$ is strongly regular, and $B$ is generated by idempotents.

In fact, by [3, Theorem 4] $S=A \oplus B$, where $A$ is strongly regular and $B$ does not contain any nonzero strongly regular ideals. $B$ is generated by idempotents by [3, Theorem 2].

By virtue of this theorem we obtain

Theorem 3.3. Let $S$ be a J-ring. Then $\bar{S}_{l}=\bar{S}_{r}$ if and only if $S$ satisfies $\left(K_{\imath}\right)$ and $\left(K_{r}\right)$.

Proof. Let $\bar{S}_{l}=\bar{S}_{r}$. By Corollary $2.3 \bar{S}_{l}$ satisfies $\left(K_{\imath}\right)$. Hence $S$ also satisfies $\left(K_{\imath}\right)$ by (ii) of Corollary 2.5. Similarly $S$ satisfies $\left(K_{r}\right)$, proving the only if part of the theorem. To see the if part let $U$ be the set of all elements $x$ of $\bar{S}_{l}$ such that $\{y \mid y \in S, x y \in S\}$ is a large right ideal of $S$. By Lemma $3.1 U$ is a right quotient ring of $S$. Let $\bar{S}_{l} \ni e=e^{2}$. Then $\left(\bar{S}_{l} e \cap S\right) \cap\left(\bar{S}_{l}(1-e) \cap S\right)=0$. By Corollary $2.4 L(S)$ is dually isomorphic to $R(S)$ and the correspondence is given by the annihilator relation. Since $\bar{S}_{l} e \cap S, \bar{S}_{l}(1-e) \cap S \in L(S)$, we have $r\left(S, \bar{S}_{l} e \cap S\right) \cup r\left(S, \bar{S}_{l}(1-e) \cap S\right)=S$ in $R(S)$. By Lemma 2.1 
$r\left(S, \bar{S}_{l} e \cap S\right)=r\left(S, \bar{S}_{l} e\right)=r\left(\bar{S}_{l}, \bar{S}_{l} e\right) \cap S=(1-e) \bar{S}_{l} \cap S$, and similarly $r\left(S, \bar{S}_{l}(1-e) \cap S\right)=e \bar{S}_{l} \cap S$. Therefore $\left((1-e) \bar{S}_{l} \cap S\right) \cup\left(e \bar{S}_{l} \cap S\right)=S$. This implies that $\left((1-e) \bar{S}_{l} \cap S\right)+\left(e \bar{S}_{l} \cap S\right)$ is a large right ideal of $S$. Evidently $e\left(\left((1-e) \bar{S}_{l} \cap S\right)+\left(e \bar{S}_{l} \cap S\right)\right)=e \bar{S}_{l} \cap S \subset S$. It follows from this that $e \in U$. Therefore we have proved that every idempotent in $\bar{S}_{l}$ is contained in $U$. By Theorem 3.2 there exists a central idempotent $f$ of $\bar{S}_{l}$ such that $\bar{S}_{l} f$ is strongly regular, and $\bar{S}_{l}(1-f)$ is generated by idempotents. Since $f \in U, U=U f \oplus U(1-f)$. As is easily seen $\bar{S}_{l} f$ is the maximal left quotient ring of $U f$. Since $\bar{S}_{l} f$ is strongly regular, $L(U f)$ is distributive by Corollary 1.3. Now by (i) of Corollary $2.5 U$ satisfies $\left(K_{l}\right)$ and $\left(K_{r}\right)$, and hence $U f$ also satisfies them by Lemma 2.6, whence $L(U f)$ and $R(U f)$ are dually isomorphic by Corollary 2.4. Thus, $R(U f)$ is distributive, and so the maximal right quotient ring of $U f$ is strongly regular by Corollary 1.3. Therefore $S_{l} f$ is a right quotient ring of $U f$ by Theorem 1.4. On the other hand, since $\bar{S}_{l}(1-f)$ is generated by idempotents, and since $U$ contains every idempotent in $\bar{S}_{l}$, it follows that $\bar{S}_{l}(1-f)=U(1-f)$. Hence $\bar{S}_{l}=\bar{S}_{l} f \oplus \bar{S}_{l}(1-f)$ is a right quotient ring of $U=U f \oplus U(1-f)$. Since $U$ is a right quotient ring of $S$, we see that $\bar{S}_{l}$ is a right quotient ring of $S$. We can prove similarly that $\bar{S}_{r}$ is a left quotient ring of $S$. Thus, we have $\bar{S}_{l}=\bar{S}_{r}$, completing the proof.

4. WR-rings and continuous rings. A ring is called a semisimple $I$-ring if every one-sided ideal contains a nonzero idempotent. By $[2$, $(4,10)]$ every semisimple $I$-ring is a $J$-ring. A ring is said to be of bounded index if there is an integer $n$ such that $x^{n}=0$ for every nilpotent element $x$. A semisimple $I$-ring is WR (=weakly reducible) if and only if every nonzero ideal contains a nonzero ideal of bounded index by [4, Theorem 9]. In [2, Theorem 5] we proved that $\bar{S}_{l}=\bar{S}_{\text {r }}$ for any semisimple WR-ring $S$. Therefore from Theorem 3.3 we obtain

TheOREM 4.1. If a semisimple I-ring $S$ is WR, then $S$ has the following property: The right (left) annihilator of a left (right) ideal $A$ is nonzero if and only if there exists a nonzero left (right) ideal $B$ such that $A \cap B=0$.

Let $S$ be a regular ring. If the lattice $L$ of principal left ideals is complete, we say that $S$ is a complete regular ring. A complete regular ring $S$ is called continuous if $L$ has the following property: $\left(\cup a_{\alpha}\right) \cap b=\bigcup\left(a_{\alpha} \cap b\right)$ and $\left(\cap a_{\alpha}\right) \cup b=\bigcap\left(a_{\alpha} \cup b\right)$ for any chain $\left(a_{\alpha}\right)$ and any element $b$ in $L$. We proved in [4, Theorem 7] that a complete regular ring is continuous if and only if it satisfies $\left(K_{\imath}\right)$ and $\left(K_{r}\right)$. Thus, by Theorem 3.3 we obtain 
ThEOREM 4.2. A complete regular ring $S$ is continuous if and only if $\bar{S}_{l}=\bar{S}_{r}$.

By [3, Theorem 3] every continuous regular ring is (both left and right) self injective if it contains no nonzero strongly regular ideals. Hence we have

THEOREM 4.3. Let $S$ be a complete regular ring, and suppose that $S$ does not contain any nonzero strongly regular ideals. Then $\bar{S}_{l}=\bar{S}_{r}$ (if and) only if $S$ is self injective, that is, $S=\bar{S}_{l}=\bar{S}_{r}$.

\section{REFERENCES}

1. R. E. Johnson, The extended centralizer of a ring over a modvle, Proc. Amer. Math. Soc. 2 (1951), 891-895.

2. Y. Utumi, On quotient rings, Osaka Math. J. 8 (1956), 1-18.

3. - On continuous regular rings and semisimple self injective rings, Canad. J. Math. 12 (1960), 597-605.

4. - On continuous regular rings, Canad. Math. Bull. 4 (1961), 63-69.

UNIVERSITY OF ROCHESTER

\section{INVERSE LIMITS OF SOLVABLE GROUPS}

ETHAN D. BOLKER

In this paper we generalize to groups of Galois type some results of $\mathrm{P}$. Hall on finite solvable groups $[1 ; 2 ; 3]$. We need, in a modified form, some results of van Dantzig: the definition of supernatural numbers (which are related to van Dantzig's universal numbers) and Theorem 5, which he proved for ordinary p-Sylow subgroups [6]. Lemmas 1 and 4 and the method of proof in Theorem 5 are due to Tate [5].

A topological group $G$ is of Galois type if it is compact and totally disconnected. In any Galois type group the open normal subgroups form a neighborhood base at the identity. Every closed subgroup is the intersection of the open subgroups containing it [4]. Whenever $M$ and $N$ are open normal subgroups of $G$ and $N \supset M$ we shall write $\phi_{N}^{M}$ for the natural homomorphism of $G / M$ onto $G / N$ (these quotient groups are finite) and $\phi_{N}$ for the natural homomorphism of $G$ onto $G / N . G$ is the inverse limit of the groups $\{G / N\}, N$ ranging over the open normal subgroups of $G$. Conversely, the inverse limit of finite groups is of Galois type. 1962.

Presented to the Society, August 30,1962; received by the editors January 31, 